\title{
Reflected and transmitted powers of electromagnetic waves through a ferrite-dielectric photonic crystal
}

\author{
Muin F. Ubeid ${ }^{\star}$, Mohammed M. Shabat \\ Department of Physics, Faculty of Science, Islamic University of Gaza, P.O. Box 108, Gaza, \\ Gaza Strip, Palestinian Authority \\ *E-mail address: mubeid@mail.iugaza.edu
}

\begin{abstract}
In this work, reflection and transmission of electromagnetic wave by a periodic ferrite-dielectric photonic crystal are investigated theoretically and numerically. The ferrite material is described and its main parameters are given in detail. After the construction of the problem, the reflection and transmission coefficients are derived in a closed form by a transfer matrix method. The reflected, transmitted, and loss powers of the crystal are calculated using these coefficients. In the numerical results the mentioned powers are computed and illustrated as a function of frequency, angle of incidence, dielectric thickness, and applied magnetic field intensity when the damping coefficient changes.
\end{abstract}

Keywords: Damping coefficient; electromagnetic waves; frequency; photonic crystal; reflection; transmission

\section{INTRODUCTION}

Photonic crystals are structures with periodically modulated dielectric constants that affects the propagation of electromagnetic waves in the same way as the periodic potential in a semiconductor crystal affects the electron motion by defining allowed and forbidden electronic energy bands [1-3]. E. Yablonovitch [4] has reviewed photonic crystals. D. J. Norris et al [5] have presented recent efforts to make three-dimensional semiconductor photonic crystals using self-assembly techniques. E. Moreno et al [6] have studied modeling of discontinuities in photonic crystal waveguide with the multipole method. O. J. Painter et al [7] have investigated two-dimensional photonic crystal defect laser.

M. F. Ubeid et al [8] have solved numerically the problem of propagation of electromagnetic waves through superconductor-dielectric photonic crystal. M. Ricci et al. [9] have investigated the dielectric losses in a superconducting photonic crystal. Arafa et al. $[10,11]$ have studied superconducting photonic crystals at microwaves, millimetrwaves, and far-infrared frequencies. 
This paper is interested in transmission and reflection of electromagnetic waves by a magnetized ferrite/dielectric photonic crystal consisting of $\mathrm{N}$ periods. We consider the photonic crystal is embedded in vacuum and a monochromatic plane electromagnetic wave is obliquely incident on it. The electric and magnetic fields are determined in each region using Maxwell's equations. Then Snell's law is applied and the boundary conditions are imposed at each interface to obtain the reflection and transmission coefficients by a transfer matrix method $[12,13]$. The reflected, transmitted, and loss powers of the structure are presented in terms of these coefficients. In the numerical analysis, the mentioned powers are calculated as a function of frequency, angle of incidence, applied magnetic field intensity and the slab thickness when the damping coefficient of ferrite changes. To check the results of the analysis used in these calculations, the conservation law of energy given in $[14,15]$ is checked and it is clear that it is satisfied for all examples.

\section{THEORY}

Consider a periodic ferrite-dielectric photonic crystal is situated between two half free spaces. The ferrite media is subjected to an external magnetic field B' along the Y axis. A perpendicular polarized plane wave (TE-polarization) in region 1 is incident on the plane $\mathrm{z}=$ 0 at some angle $\theta$ relative to the normal to the boundary, see Fig. 1. In this figure, the letters $\mathrm{F}$ and $\mathrm{D}$ stand for ferrite and dielectric, $\mathrm{d}_{\mathrm{F}}$ and $\mathrm{d}_{\mathrm{D}}$ are the thicknesses of ferrite and dielectric layers in each period, $\mathrm{d}=\mathrm{d}_{\mathrm{F}}+\mathrm{d}_{\mathrm{D}}, \mathrm{N}$ is the number of periods, $2 \mathrm{~N}+2$ is the number of regions, the numbers $1,2,3,4, \ldots 2 \mathrm{~N}+2$ stand for the region order.
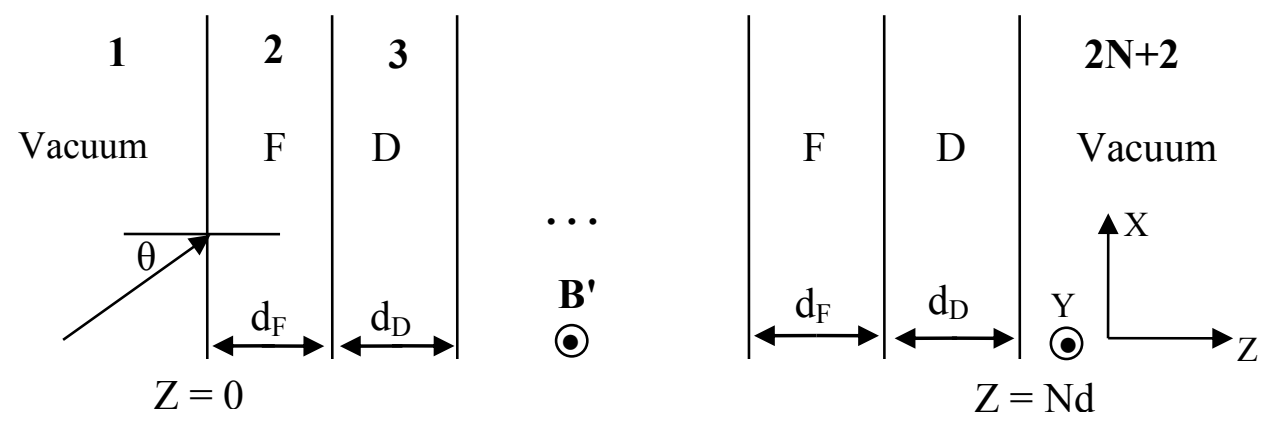

Figure 1. Geometry of the proposed ferrite-dielectric photonic crystal.

The permeability of ferrite is a tensor characteristic with effective permeability of the form $[16,17]$ :

$$
\mu_{F}=\frac{\eta^{2}+\mathrm{K}^{2}}{\eta}
$$

where,

$$
\eta=1+\frac{\omega_{m}\left(\omega_{o}-i \alpha \omega\right)}{\left(\omega_{o}-i \alpha \omega\right)^{2}-\omega^{2}}, \mathrm{~K}=-\frac{i \omega_{m} \omega}{\left(\omega_{o}-i \alpha \omega\right)^{2}-\omega^{2}}, \omega_{o}=\gamma B^{\prime}, \omega_{m}=\gamma M
$$

$\gamma$ is the gyromagnetic ratio, $B^{\prime}$ is the intensity of the applied magnetic field, $M$ is the saturation magnetization, $\omega$ is the angular frequency, $\alpha$ is the damping coefficient of 
precession (damping coefficient) and $i=\sqrt{-1}$. The permittivity tensor of the ferrite has a diagonal form with components $\varepsilon_{i i}=\varepsilon_{\mathrm{F}}$.

The transverse components of the electric fields of the incident and reflected waves in each region of Fig. 1 are $[18,19]$ :

$$
E_{m y}=\left(A_{m} e^{i k_{m z} z}+B_{m} e^{-i k_{m z} z}\right) e^{i\left(k_{m x} x-\omega t\right)}
$$

To find the corresponding magnetic field $\vec{H}$, we start with Maxwell's equation [18]: $\vec{\nabla} \times \vec{E}=-\frac{\partial \vec{B}_{o}}{\partial t}$, substituting $\vec{B}_{o}=\mu \vec{H}$ and solving for $\vec{H}$. That is for the ferrite layer:

$$
H_{F m x}=\frac{1}{\omega \mu_{o}}\left[\left(A_{m} k_{c} e^{i k_{m z} z}+B_{m} k_{b} e^{-i k_{m z} z}\right)\right] e^{i\left(k_{m x} x-\omega t\right)}
$$

with,

$$
k_{c}=\frac{k_{m x}}{\mu_{v}}-\frac{k_{m z}}{\mu_{f}}, k_{b}=\frac{k_{m x}}{\mu_{v}}+\frac{k_{m z}}{\mu_{f}}, \mu_{v}=\frac{\eta^{2}+\mathrm{K}^{2}}{-\mathrm{K}}
$$

for the dielectric layer (as well as the initial and final media):

$$
H_{m x}=\frac{1}{\mu_{o} \omega}\left[\left(-A_{m} k_{m z} e^{i k_{m z} z}+B_{m} k_{m z} e^{-i k_{m z} z}\right)\right] e^{i\left(k_{m x} x-\omega t\right)}
$$

where $A_{m}$ and $B_{m}$ are the amplitudes of the forward and backward traveling waves in the mth region, $k_{m}=n_{m} \omega / c$ is the wave number of the medium and $n_{m}=\sqrt{\varepsilon_{m} \mu_{m}}$ is the refractive index of it $(\mathrm{m}=1,2 \mathrm{~N}+1$ for initial and final media respectively, $\mathrm{m}=2,4,6, \ldots 2 \mathrm{~N}$ for ferrite layers, $\mathrm{m}=3,5,7 \ldots 2 \mathrm{~N}+1$ for dielectric layers).

The subscripts $\mathrm{x}$ and $\mathrm{z}$ represent the $\mathrm{x}$ - and $\mathrm{z}$-components of the related wave number, respectively. These components are:

$k_{m x}=k_{x}=\frac{\omega n_{1}}{c} \sin \theta \equiv$ Snell's law

$$
k_{m z}=\frac{\omega}{c} \sqrt{n_{m}^{2}-n_{1}^{2} \sin ^{2} \theta}
$$

Now since the structure is infinite and periodic consisting of alternate ferrite and dielectric layers, the boundary conditions can be imposed.

These conditions imply that, the tangential electric field component and the normal component of the corresponding magnetic field are continuous at the boundaries between the layers.

Using the method of the transmission matrix (which relates the fields at the beginning and the end of the period of the structure) and applying the Floquet theorem, which takes into account the periodicity of the structure, we arrive at the dispersion relation: 


$$
\cos \bar{k} d=\cos k_{2 z} d_{F} \cos k_{3 z} d_{D}-\frac{1}{2}\left[\begin{array}{l}
\frac{k_{2 z}}{k_{3 z}} \frac{1}{\mu_{f}}+\frac{k_{3 z}}{k_{2 z}} \mu_{f} \\
-\frac{k_{x}{ }^{2}}{k_{2 z} k_{3 z} \mu_{f}}\left(\frac{K}{\eta}\right)^{2}
\end{array}\right] \sin k_{2 z} d_{F} \sin k_{3 z} d_{D}
$$

The components of the transfer matrix $\hat{m}$ for one period of the ferrite-dielectric photonic crystals are[12]:

$$
\begin{gathered}
m_{11}=\cos k_{2 z} d_{F} \cos k_{3 z} d_{D}-\frac{k_{3 z}}{k_{2 z}} \mu_{F} \sin k_{2 z} d_{F} \sin k_{3 z} d_{D} \\
+i \frac{k_{x}}{k_{2 z}} \frac{K}{\eta} \sin k_{2 z} d_{F} \cos k_{3 z} d_{D}, \\
m_{12}=i \frac{\omega}{c}\left(\frac{\left.\frac{1}{k_{3 z}} \cos k_{2 z} d_{F} \sin k_{3 z} d_{D}+\frac{\mu_{F}}{k_{2 z}} \sin k_{2 z} d_{F} \cos k_{3 z} d_{D}+\right)}{i \frac{k_{x}}{k_{2 z} k_{3 z}} \frac{K}{\eta} \sin k_{2 z} d_{F} \sin k_{3 z} d_{D}}\right) \\
m_{21}=i \frac{c}{\omega}\left(\frac{1}{\mu_{F}}\left(-\frac{k_{x}^{2}}{k_{2 z}}\left(\frac{K}{\eta}\right)^{2}+k_{2 z}\right) \sin k_{2 z} d_{F} \cos k_{3 z} d_{D}+k_{3 z} \cos k_{2 z} d_{F} \sin k_{3 z} d_{D}\right) \\
-i k_{x} \frac{k_{3 z}}{k_{2 z}} \frac{K}{\eta} \sin k_{2 z} d_{F} \sin k_{3 z} d_{D} \\
m_{22}=-\frac{1}{\mu_{F}} \frac{1}{k_{3 z}}\left(-\frac{k_{x}^{2}}{k_{2 z}}\left(\frac{K}{\eta}\right)^{2}+k_{2 z}\right) \sin k_{2 z} d_{F} \sin k_{3 z} d_{D}+\cos k_{2 z} d_{F} \cos k_{3 z} d_{D} \\
-i \frac{k_{x}}{k_{2 z}} \frac{K}{\eta} \sin k_{2 z} d_{F} \cos k_{3 z} d_{D} .
\end{gathered}
$$

Raising the transfer matrix $\widehat{m}$ for one period to the N-th power $\left(\widehat{S}=(\widehat{m})^{N}\right)[12]$ :

$$
\begin{gathered}
S_{11}=m_{11} \frac{\sin N \bar{k} d}{\sin \bar{k} d}-\frac{\sin (N-1) \bar{k} d}{\sin \bar{k} d}, \\
S_{12}=m_{12} \frac{\sin N \bar{k} d}{\sin \bar{k} d}, \\
S_{21}=m_{21} \frac{\sin N \bar{k} d}{\sin \bar{k} d}, \\
S_{22}=m_{22} \frac{\sin N \bar{k} d}{\sin \bar{k} d}-\frac{\sin (N-1) \bar{k} d}{\sin \bar{k} d} .
\end{gathered}
$$

Using the boundary conditions for tangential components of the electromagnetic field at $\mathrm{Z}=0$ and $\mathrm{Z}=\mathrm{Nd}$, enable us to express the reflection and transmission coefficients $\mathrm{R}^{\prime}$ and $\mathrm{T}^{\prime}$, respectively [12]: 


$$
\begin{aligned}
& R^{\prime}=\frac{\frac{\omega}{c} k_{1 z} S_{11}-\left(\frac{c}{\omega}\right)^{2} k_{1 z} k_{(2 N+2) z} S_{12}+S_{21}-\frac{c}{\omega} k_{(2 N+2) z} S_{22}}{\frac{c}{\omega} k_{1 z} S_{11}-\left(\frac{c}{\omega}\right)^{2} k_{1 z} k_{(2 N+2) z}-S_{21}+\frac{c}{\omega} k_{(2 N+2)} S_{22}} \\
& T^{\prime}=\frac{2 \frac{\omega}{c} k_{1 z} \exp \left(-i k_{(2 N+2) z} N d\right)}{\frac{c}{\omega} k_{1 z} S_{11}-\left(\frac{c}{\omega}\right)^{2} k_{1 z} k_{(2 N+2) z}-S_{21}+\frac{c}{\omega} k_{(2 N+2)} S_{22}}
\end{aligned}
$$

The reflected and transmitted powers $\mathrm{R}$ and $\mathrm{T}$, respectively of the structure can be expressed as:

$$
\begin{aligned}
& \mathrm{R}=\mathrm{R}^{\prime} \mathrm{R}^{\prime *}, \\
& \mathrm{~T}=\mathrm{T}^{\prime} \mathrm{T}^{\prime *}
\end{aligned}
$$

where $\mathrm{R}^{\prime *}$ and $\mathrm{T}^{\prime *}$ are the complex conjugates of $\mathrm{R}^{\prime}$ and $\mathrm{T}^{\prime}$, respectively. The law of conservation of energy is given by $[14,15]$ :

$$
\mathrm{R}+\mathrm{T}=1-\mathrm{P}_{\text {loss }}
$$

where $\mathrm{P}_{\text {loss }}$ is the loss power due to losses in the ferrite layers.

\section{NUMERICAL RESULTS}

In this section, the reflected, transmitted, and loss powers of the ferrite-dielectric photonic crystal are calculated as a function of frequency, angle of incidence, external magnetic intensity, and dielectric thickness for changing values of the damping coefficient.

In the calculations the following parameters are used as in [20]:

$\mathrm{M}=3.5 \times 10^{5} \mathrm{~A} / \mathrm{m}, \mathrm{B}^{\prime}=4 \times 10^{4} \mathrm{~A} / \mathrm{m}, \gamma=2 \pi 3.518 \times 10^{-5} \mathrm{GHz} /(\mathrm{A} / \mathrm{m}), \varepsilon_{\mathrm{F}}=4$.

The dielectric layers are selected to become silicon-Si (permittivity 12.25 and permeability 1). The operating frequency is assumed to be $\mathrm{f}_{0}=100 \mathrm{GHz}$.

The other parameters are selected to be: the ferrite thickness $d_{F}=\lambda / 4$, the dielectric thickness $d_{D}=\lambda / 8$, where $\lambda$ is the wavelength calculated at the operating frequency, the number of periods $\mathrm{N}=5$, and the angle of incidence $\theta=30^{\circ}$. Two cases of the ferrite are considered, loss-less case $(\alpha=0)$ and loss case $(\alpha \neq 0)$.

Figure 2 shows the reflected, transmitted, and loss power as a function of frequency for three different values of the damping coefficient $(\alpha=0, .1, .2)$.

The frequency is changed between $10 \mathrm{GHz}$ and $200 \mathrm{GHz}$. It can be seen that, the powers have an oscillatory behaviors when the frequency changes. The reflected power (the transmitted and loss powers) is maximum (minimum) at the frequency bands $66-84 \mathrm{GHz}$ and 142-172 GHz. 


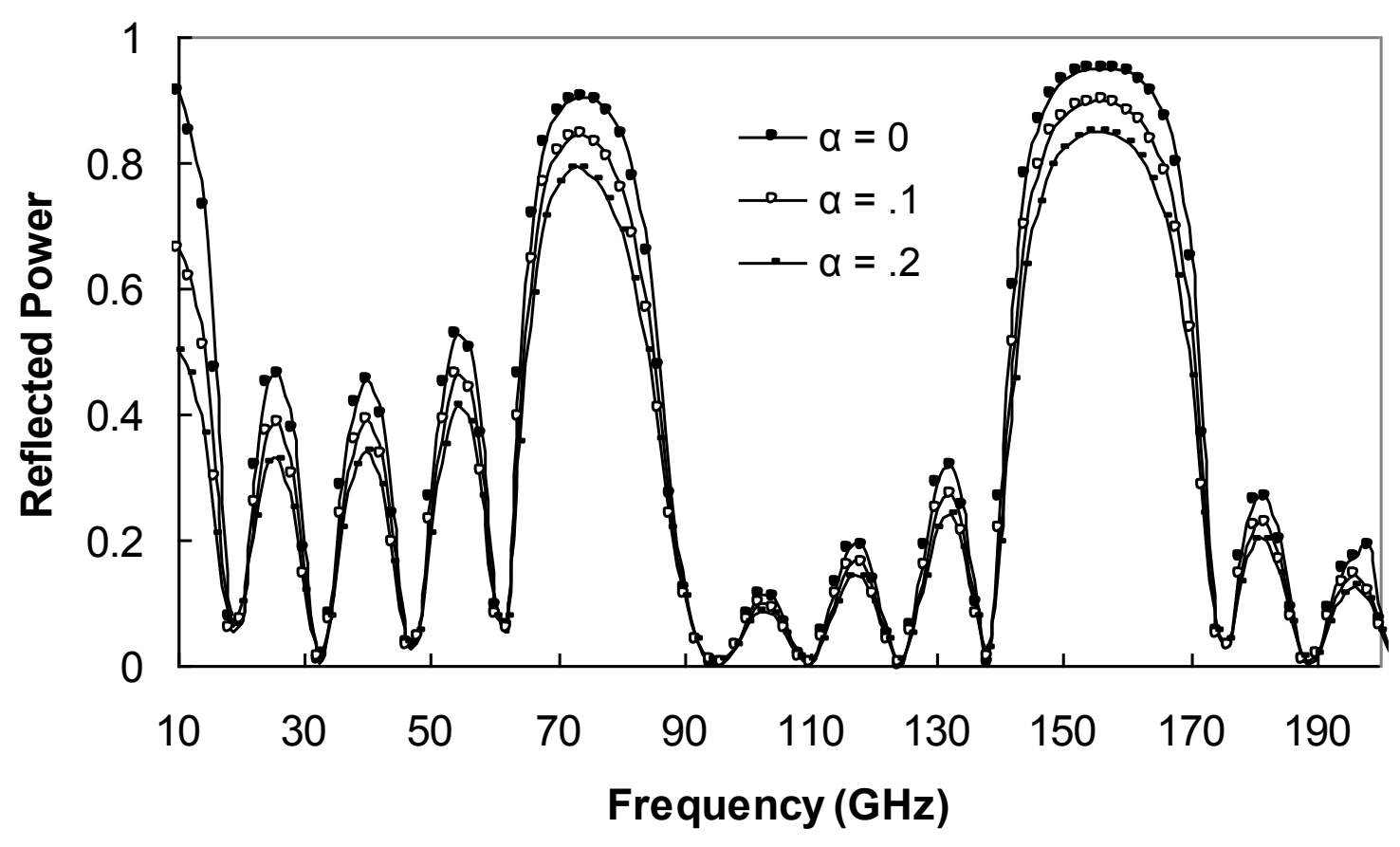

(a)

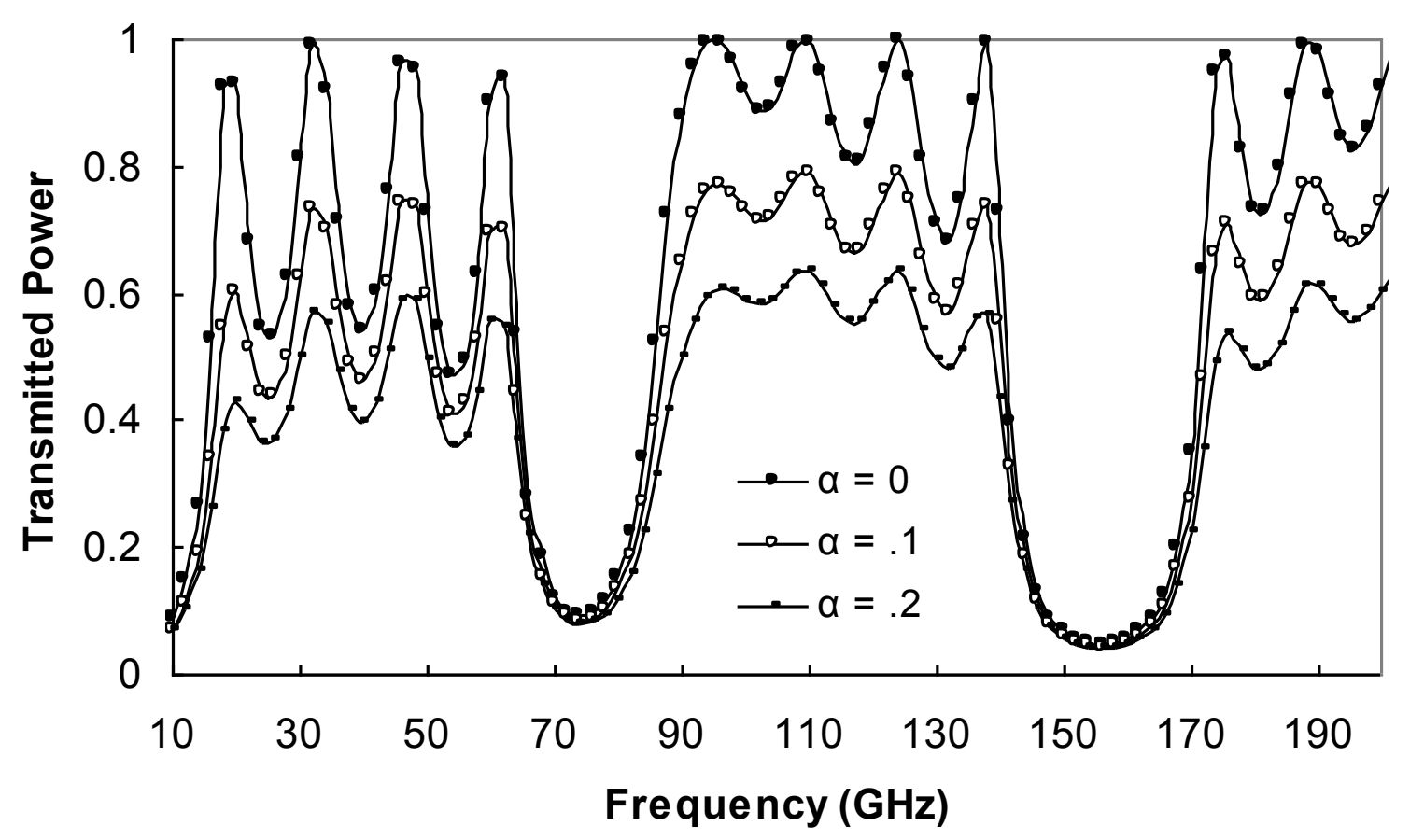

(b)

Figure 2. The reflected, transmitted, and loss powers as a function of the frequency. 


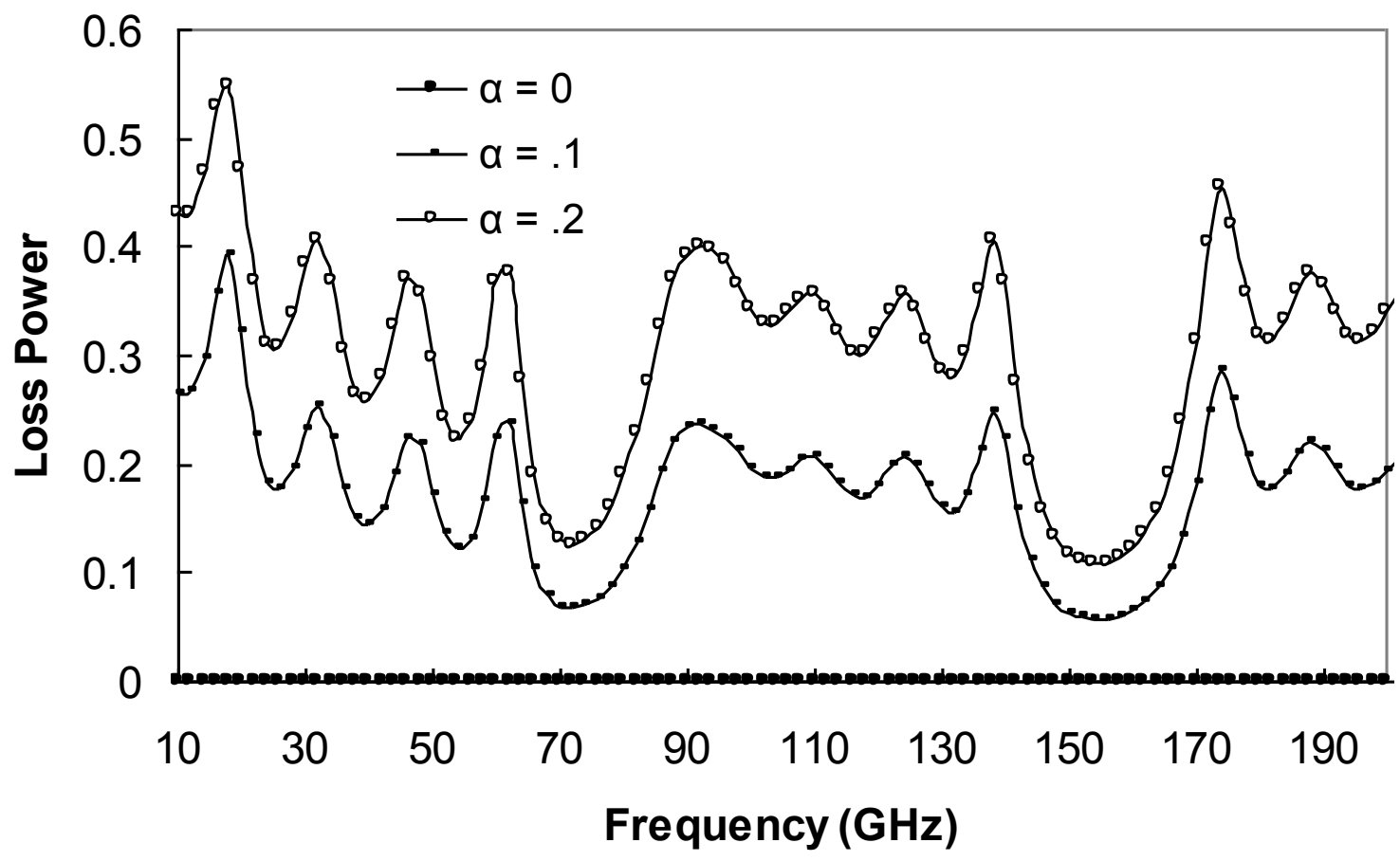

(c)

Figure 2 (continue). The reflected, transmitted, and loss powers as a function of the frequency.

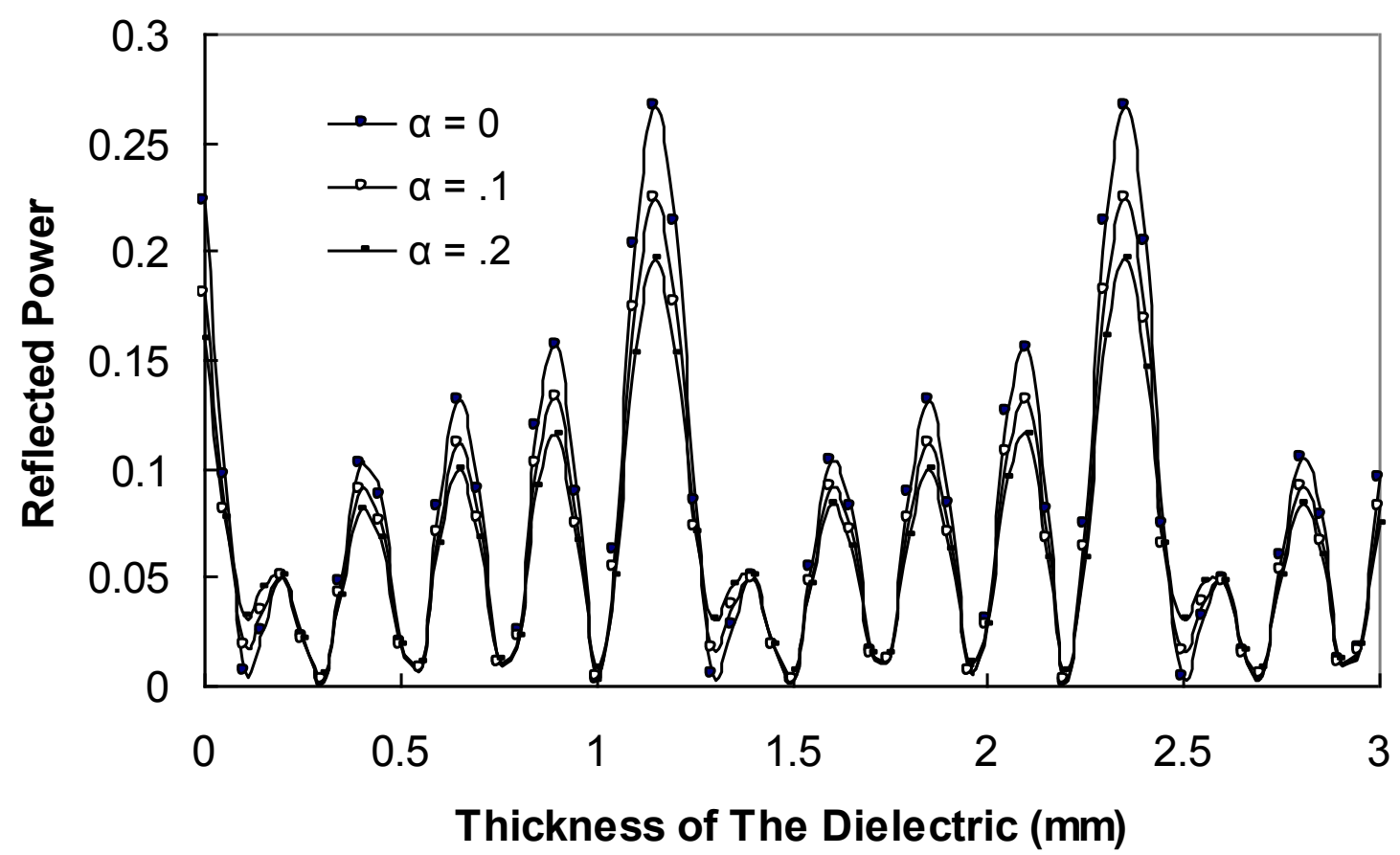

(a)

Figure 3. The reflected, transmitted, and loss powers against dielectric thickness. 


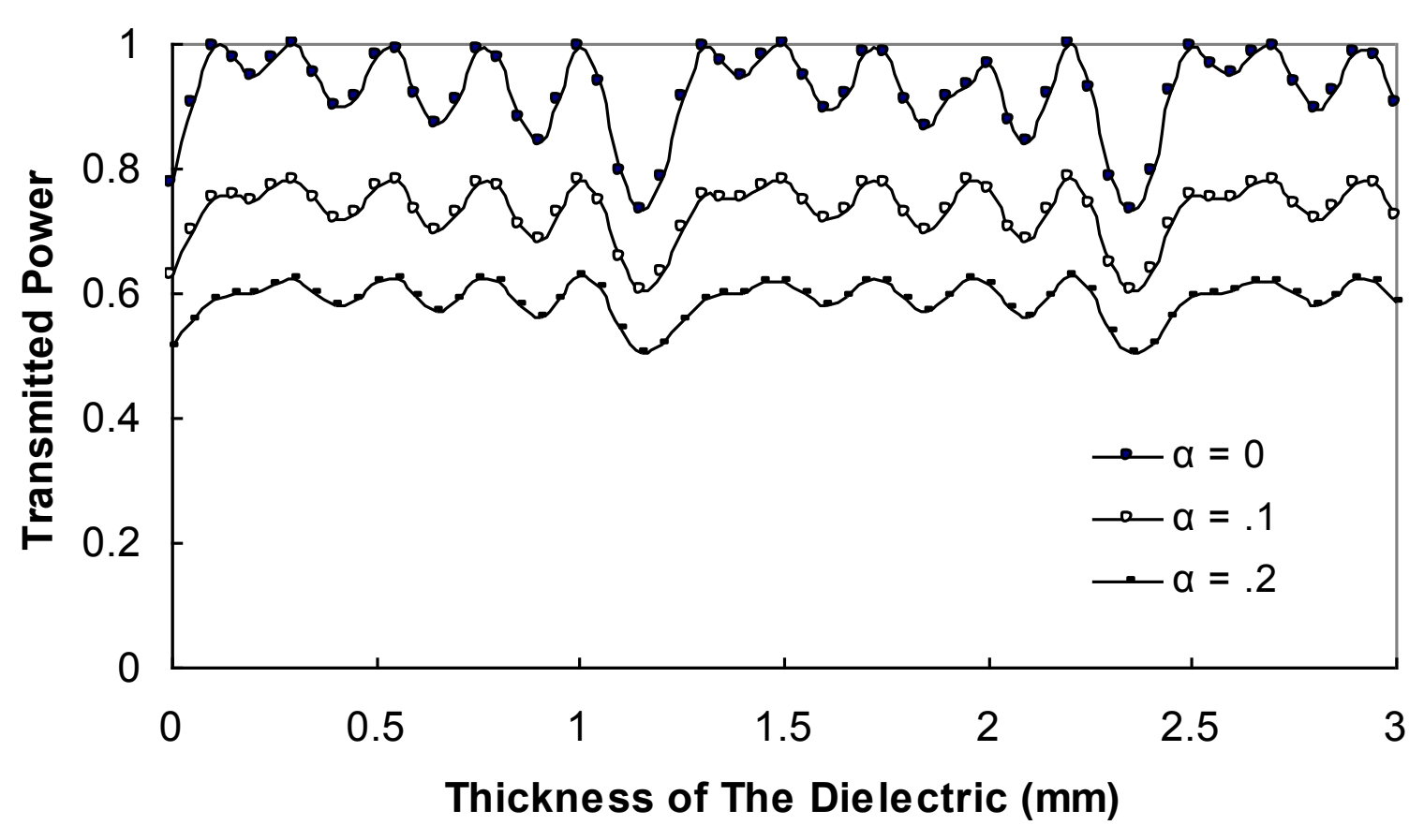

(b)

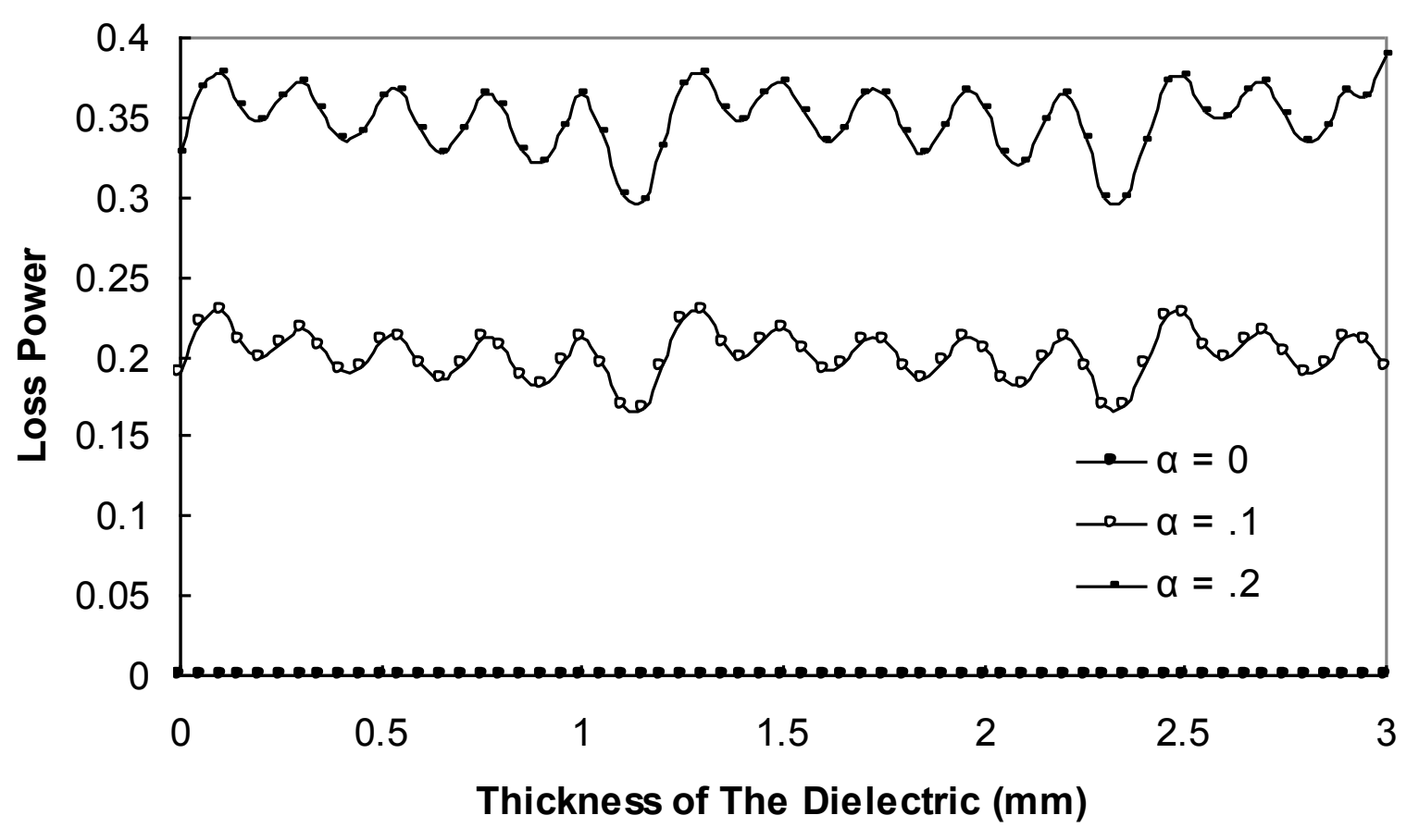

(c)

Figure 3 (continue). The reflected, transmitted, and loss powers against dielectric thickness. 
Figure 3 depicts the reflected, transmitted and loss powers against the thickness of the dielectric when the damping coefficient changes $(\alpha=0, .1, .2 \mathrm{GHz})$ at the frequency 100 GHz. The dielectric thickness is changed from zero to $\lambda(3 \mathrm{~mm})$. As it confirmed from the figure, the powers vary periodically with dielectric thickness. The reflected power (the transmitted and loss powers) is maximum (minimum) at the thickness $1.15 \mathrm{~mm}$. It is observed that, this maximum (minimum) value appears every $1.15 \mathrm{~mm}$ thickness of the dielectric.

Figure 4 illustrates the variation of the reflected, transmitted, and loss powers with the angle of incidence for $16 \mathrm{GHz}$ frequency under three values of the damping coefficient. The angle of incidence is changed between $0^{\circ}$ and $90^{\circ}$ to realize all possible angles of incidence. Clearly the reflected power increases while the transmitted and loss powers decrease with the angle of incidence. At $90^{\circ}$ the reflected (the transmitted and loss) powers is maximum (minimum) at that angle for any value of dissipation factor. The role of the dissipation factor is clear at angles below $90^{\circ}$. The reflected and transmitted powers decrease while the loss power increases with the dissipation factor for any angle below $90^{\circ}$.

Figure 5 demonstrates the effect of the external magnetic field intensity on the reflected and transmitted powers for two values of the frequency of the incident waves $(f=5.2,6.2$ $\mathrm{GHz}$ ). For simplicity, the damping coefficient is selected to be zero. The applied magnetic field intensity is changed from zero $\mathrm{A} / \mathrm{m}$ to $2.0 \times 10^{5} \mathrm{~A} / \mathrm{m}$. It is noticed from the figure that, there is a drop (rise) in the reflected (transmitted) power at specified value of the applied magnetic field for each frequency. Moreover, the symmetry shifts to the right with the applied magnetic fields when the frequency increased.

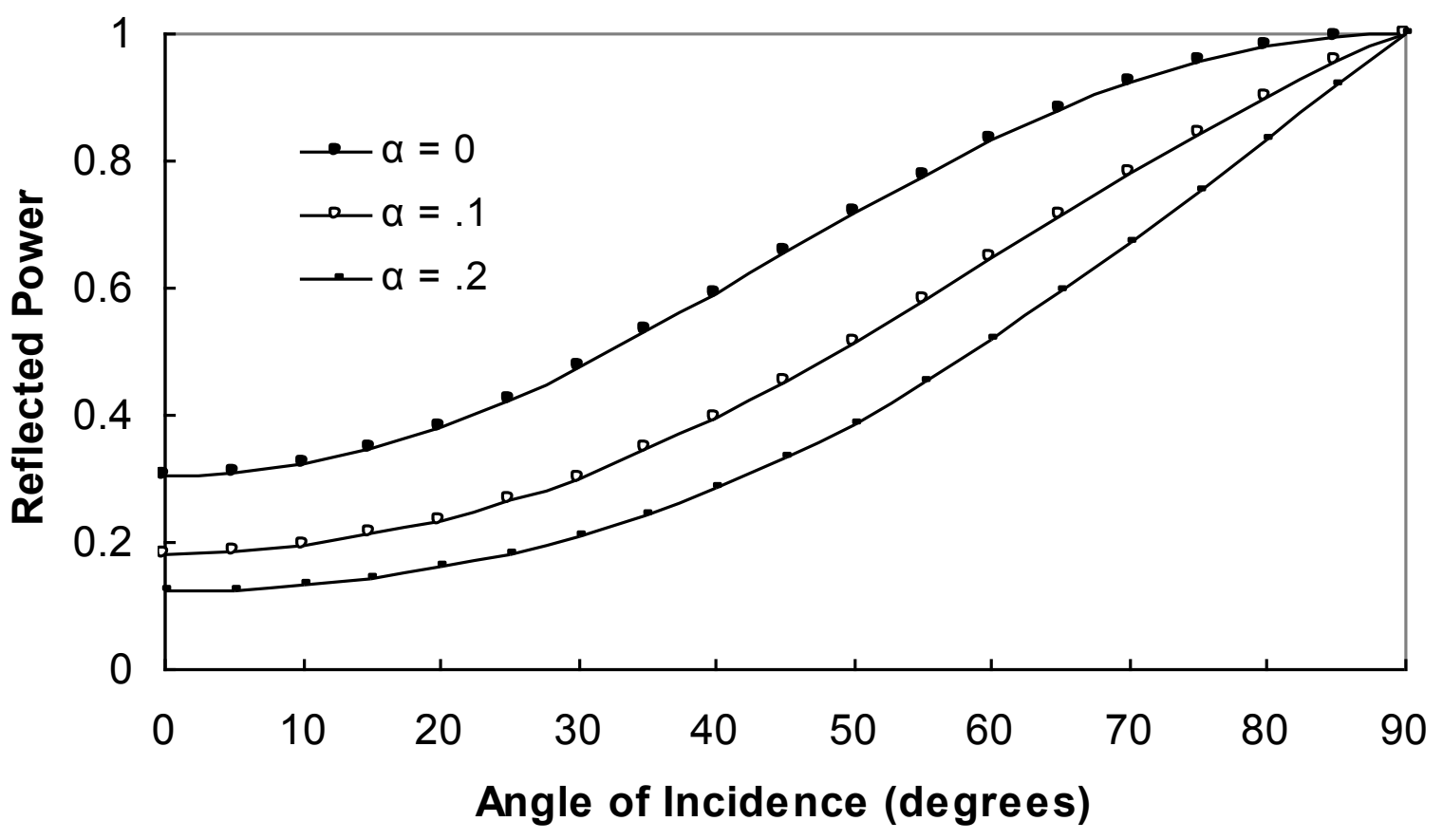

(a)

Figure 4. The reflected, transmitted and loss powers variation with the angle of incidence. 


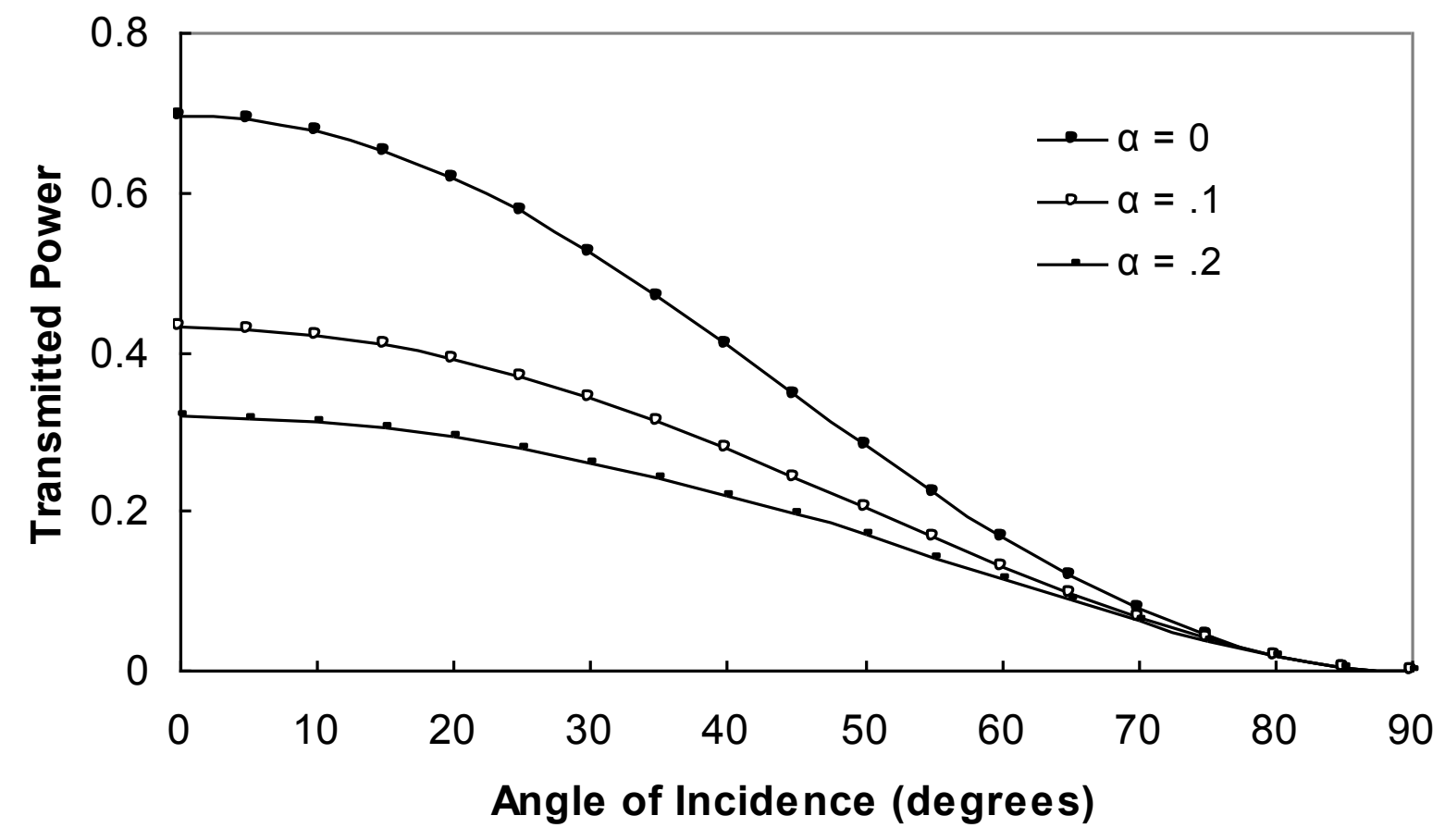

(b)

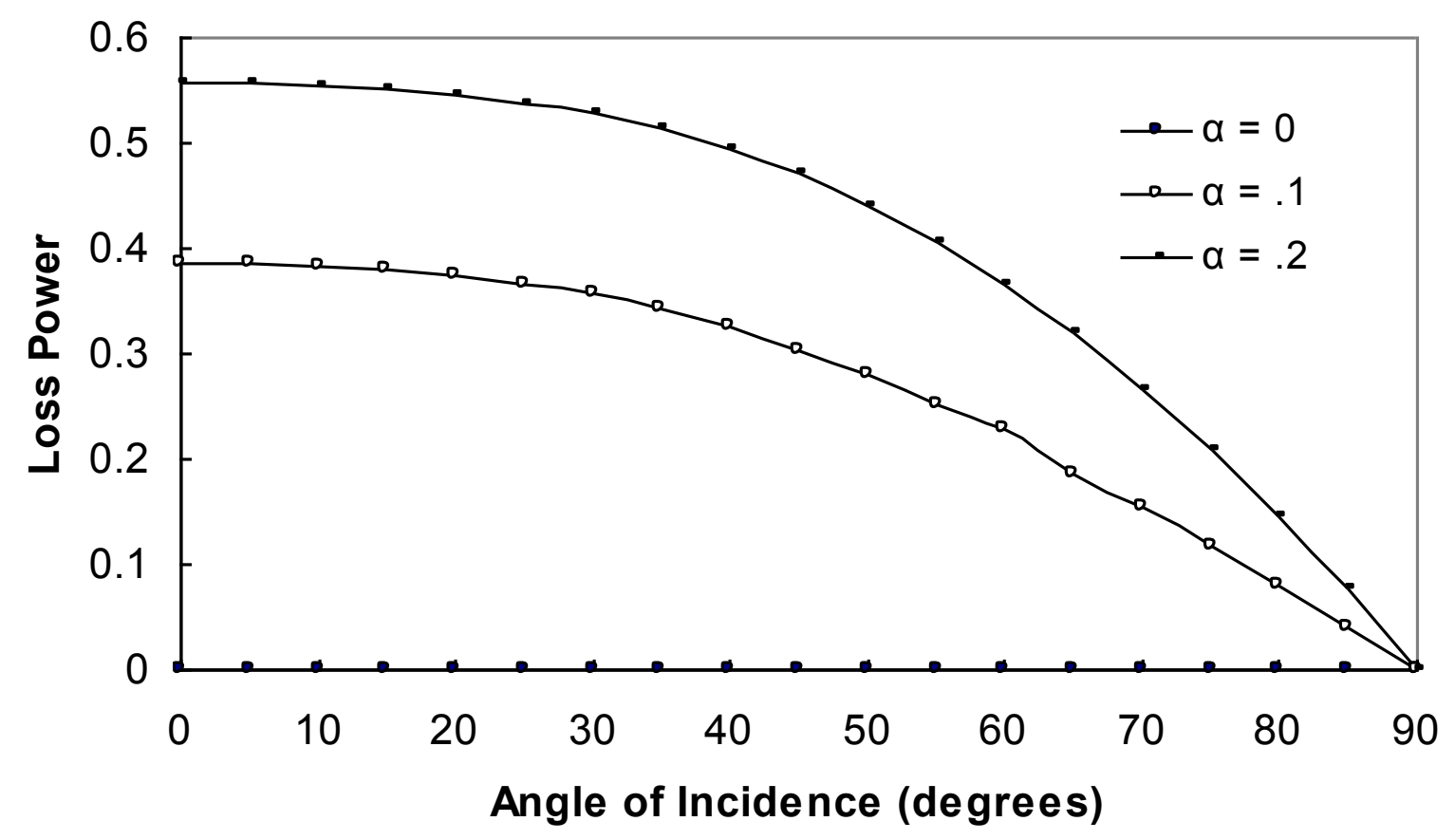

(c)

Figure 4 (continue). The reflected, transmitted and loss powers variation with the angle of incidence. 


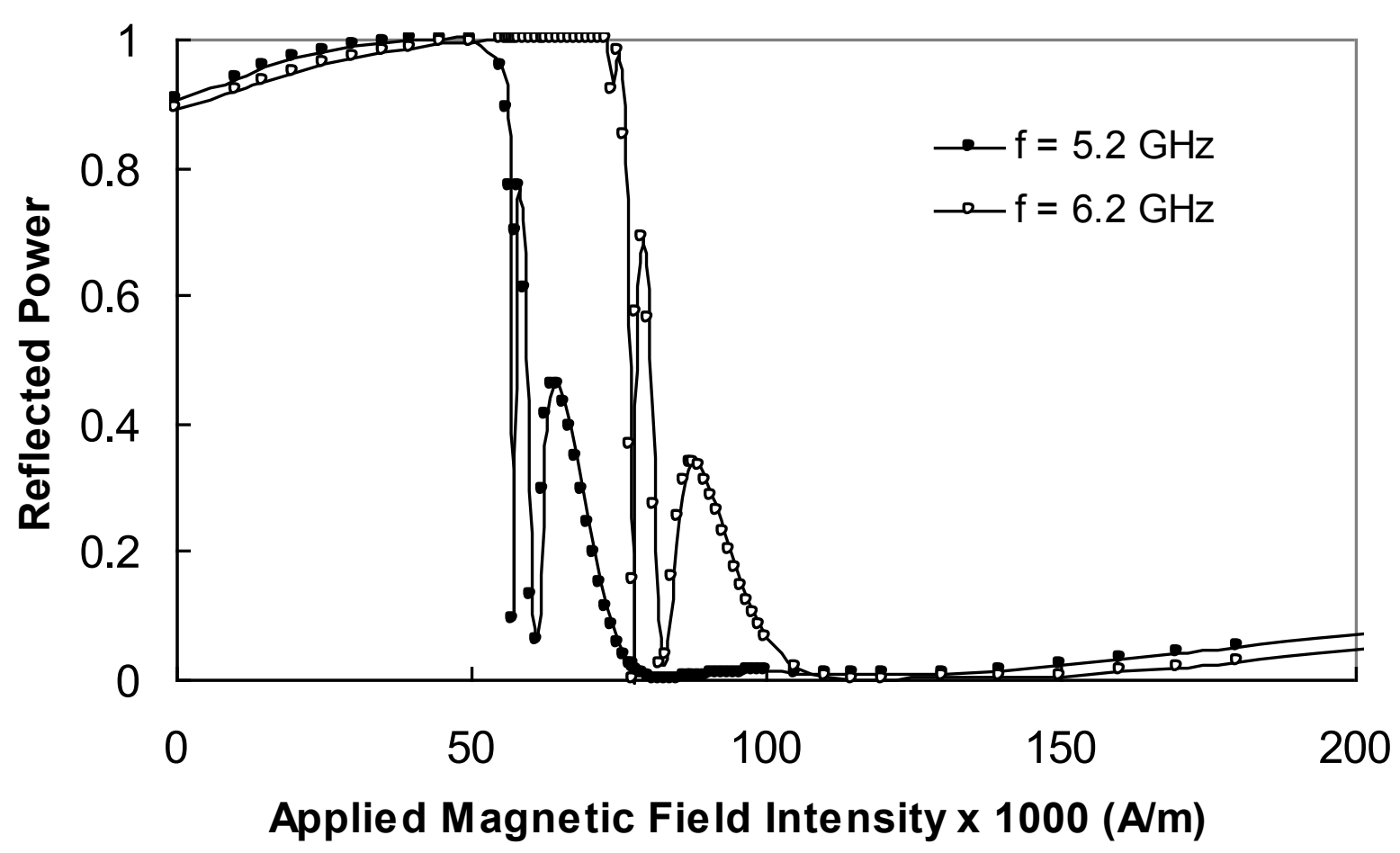

(a)

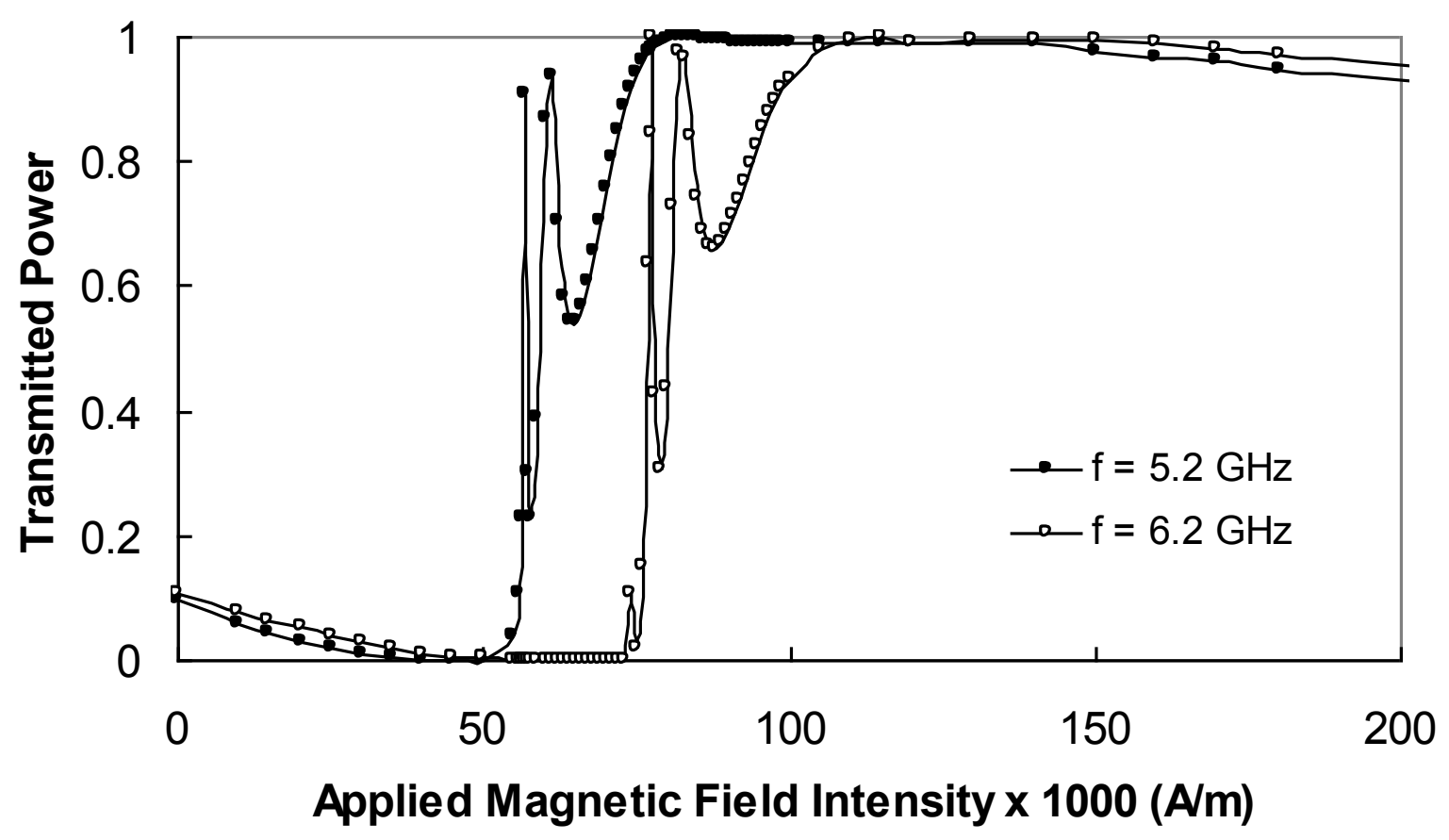

(b)

Figure 5. The reflected and transmitted powers versus the external applied magnetic field intensity. 


\section{CONCLUSIONS}

In this paper, the reflection and transmission characteristics of the electromagnetic radiation propagation through a ferrite/dielectric (Silicon-Si) photonic crystal are studied in detail with the effect of the damping coefficient. The required equations for the electric and magnetic fields in each region are derived by Maxwell's equations. Then Snell's law is applied and the boundary conditions are imposed to calculate the reflection and transmission coefficients of the crystal. Transfer matrix method is used to solve the problem of electromagnetic wave propagation through the crystal to obtain the reflected, transmitted, and loss powers. Finally, the mentioned powers as a function of frequency, angle of incidence and the dielectric thickness etc. are studied numerically to observe the effect of the damping coefficient on them. As it can be seen from the theoretical and the numerical results, if the damping coefficient changes, the characteristic of the powers will be affected by this change. Numerical examples are already presented to illustrate the paper idea and to prove the validity of the obtained results. Moreover the law of conservation of energy is satisfied throughout the performed computations for all examples.

The results obtained in this paper can be helpful to design new devices, apparatus, components at the millimeter wave, optical, and microwave regimes. Furthermore, these results open a way to think how the availability of the damping coefficient will change the functionality of a device with ferrite/dielectric photonic crystal.

\section{References}

[1] J. D. Joannopoulos, R. D. Meade, J. N. Winn, Photonic Crystals, Princeton Univ. Press (1995).

[2] J. D. Joannopoulos, P. R. Villeneuve, S. Fan, Nature 386 (1997)143-149.

[3] Y. A. Vlasov, X. Z. Bo, J. C. Sturm, D. J. Norris, Nature 414 (2001) 289-293.

[4] E. Yablonovitch, Photonic crystal: semiconductors of light, Scientific American, 12 (2001).

[5] D. J. Norris, Yu. A. Vlasov, Advanced materials 3 (2001) 371-376.

[6] E. Moreno, D. Erni, Ch. Hafner, Phys. Rev. E, 66 (2002) 036618.

[7] O. J. Painter, A. Husain, A. Scherer, J. D. O’Brien, I. Kim, P. D. Dapkus, J. Lightwave Techn. 17 (1999) 2082-2089.

[8] M. F. Ubeid, M. M. Shabat, M. O. Sid-Ahmed, Lecture Notes on Photonics and Optoelectronics 1 (2013) 35-39.

[9] M. Ricci, N. Qrloff, S. M. Angale, App. Phys. Lett. 87 (2005) 034102-(1-3).

[10] A. H. Aly, Materials Chemistry and Physics 115 (2009) 391-394.

[11] A. H. Aly, Heng-Tung Hsu, Tzong-Jer Yang, Chien-Jang Wu, C. K. Wangbo, J. of Applied Physics, 105 (2009) 0839(17-23).

[12] O. V. Shramkova, Progress In Electromagnetics Research $M 7$ (2009) 71-85.

[13] C. Sabah, S. Uckun, Journal of Optoelectronics and Advanced Materials (JOAM) 9 (2007) 2480-2484. 
[14] M. F. Ubeid, M. M. Shabat, M. O. Sid-Ahmed, International Letters of Chemistry, Physics and Astronomy 3 (2013) 1-11.

[15] D. D. Stancil, A Prabhakar, Spin Waves, New York: Springer (2009).

[16] G. Dewar, J. Appl. Phys. 97 (2005) 10Q101: 1-3.

[17] G. Dewar, Proceeding of SPIE (2003) 5218: 140-144

[18] R.A. Shelby, Thesis (PhD.), University of Calefornia, San Diego, Microwave Experiments with Left-Handed Materials, Bell and Howell Information and Learning Company (2001).

[19] M. Augustine, S. Mathew, V. Mathew, Int. J. Milliwaves 29 (2008) 534-544.

[20] M. F. Ubeid, M. M. Shabat, M. O. Sid-Ahmed, Journal of Nano- and Electronic Physics 4 (2012) 01009(1-4). 